\title{
Winkelstabile Plattenosteosynthese an der Tibia
}

\author{
Yves Pascal Acklin, Christoph Sommer
}

\section{Zusammenfassung}

Der zunehmende Einsatz von winkelstabilen Implantaten erleichtert die Plattenosteosynthese an der Tibia. Einerseits wird somit eine gute Plattenverankerung in osteoporotischem Knochen gewährleistet. Andererseits erleichtert die stabile Schrauben-Platten-Verbindung die Anwendung einer minimalinvasiven Technik und ermöglicht die unilaterale Versorgung von instabilen Gelenkfrakturen.

\section{Angle-Stable Implants for Plating the Tibia}

The increasing use of angle-stable implants facilitates the plating of the tibia. On the one hand, a good anchorage of the plate is provided in osteoporotic bone. On the other hand, the stable screw-plate interface facilitates the application of a minimally invasive technique in fracture fixation and enables unilateral fixation of unstable articular fractures.

\section{Einleitung}

Das Aufkommen der winkelstabilen Implantate erleichtert die Plattenosteosynthesen bei komplexen und gelenksnahen Frakturen der Tibia [1]. Die Plattenentwicklung zeigte nicht nur Erfolge bei osteoporotischen Frakturen, sondern begünstigte auch minimalinvasive Operationstechniken.

Grundsätzlich zeigen winkelstabile Implantate mehrere Vorteile bei der Versorgung von Tibiafrakturen. Durch die Winkelstabilität der Schrauben ist ein Pull-out der Platte im herkömmlichen Sinne vor allem bei osteoporotischen Frakturen deutlich seltener. Die winkelstabilen Schrauben lassen sich im Gegensatz zu den konventionellen Schrauben durch eine Plattenabscherbewegung nicht axial aus dem Kochen entfernen und vergrößern die Haltekraft [2].

Erst die winkelstabilen Implantate verhindern eine Fraktursinterung auf der plattenfernen Seite mit sekundärer Achsenabweichung und erlauben deshalb in vielen Fällen eine unilaterale Versorgung

OP-JOURNAL 2012; 28: 144-147

(c) Georg Thieme Verlag KG Stuttgart · New York DOI http://dx.doi.org/10.1055/s-0032-1315338 von komplexen Gelenksfrakturen mit metaphysärer Trümmerzone.

Und letztendlich erleichtern die winkelstabilen Implantate die minimalinvasive Plattenosteosynthese (MIPO) vornehmlich zur Überbrückung einer metaphysären Frakturzone. Mit dem Prinzip des internen Fixateurs muss die Platte nicht mehr vollständig dem Knochen anliegen und ermöglicht so eine Fixation, ohne die Fraktur zu verwerfen.

\section{Winkelstabile Osteosyntheseverfahren bei proximalen Tibiafrakturen}

In der Mehrzahl der Fälle haben proximale Tibiafrakturen eine intraartikuläre Komponente. Seltener kommen reine metaphysäre Frakturen oder Kombinationen von intra- und metaphysären Frakturen vor. Die intraartikuläre Frakturkomponente benötigt eine direkte Visualisierung zur anatomischen Wiederherstellung der Gelenkskongruenz einerseits über einen Arthrotomie- oder andererseits über eine Arthroskopie. Die Schraubenwahl folgt dem Frakturtyp, wobei die Unterscheidung in partiell artikulär (B-Typ-Frakturen) und komplett artikulär (C-Typ-Frakturen) entscheidend ist. B-Typ-Frakturen benötigen eine Plattenosteosynthese mit Abstütz- respektive Antigleitfunktion. Winkelstabili- tät ist prinzipiell nicht nötig, da die proximalen Schrauben an der intakten frakturabgewandten Seite und in der Platte Halt finden (Abb. 1). Dies gilt insbesondere bei jungen Patienten mit guter Knochenqualität. Bei osteoporotischen Frakturen ist die Winkelstabilität aber auch bei diesen „einfacheren“ B-Frakturen von Vorteil, da sich die Schrauben durch die Verblockung im Plattenloch nicht mehr lockern können (Abb.2). Eine C-Typ-Fraktur hingegen benötigt eine mediolaterale Doppelplattenosteosynthese oder eine unilaterale Versorgung mittels winkelstabilem Implantat. Damit kann eine Angulierung der Schrauben und somit eine konsekutive Varisierung resp. Valgisierung der Fraktur vermieden werden (Abb.3). Für C-Typ-Frakturen konnte gezeigt werden, dass ein Versagen der Osteosynthese bei Doppelplattenosteosynthese erst bei einer Belastung von über $1000 \mathrm{~N}$ erfolgt. Das Versagen der Osteosynthese bei unilateraler Versorgung mittels winkelstabilen Implantaten liegt mit 500-700 N zwar unter diesem Wert, jedoch noch deutlich über den postoperativ erlaubten 10$15 \mathrm{~kg}$ [3]. Die erfolgreiche unilaterale Versorgung von C-Frakturen mit metaphysärer Trümmerzone bedingt aber einerseits eine kräftige Platte (z.B. LCP-PLT) und andererseits eine gute Patientencompliance mit Einhalten der empfohlenen Abrollbelastung während sicher 6 Wochen. Eine $\mathrm{zu}$ frühe Vollbelastung könnte zu einer Verbiegung der Platte führen. Diese Regel gilt nicht nur für die proximale, sondern für die gesamte Tibia.

Winkelstabile Implantate ermöglichen die unilaterale Fixation von Gelenksfrakturen.

Luxationstypfrakturen, welche in ihrer Verletzungsentität mit immer vorhandener ligamentärer Zusatzverletzung eine Spezialstellung einnehmen, benötigen meistens eine Antigleitplatte [4]. Somit 

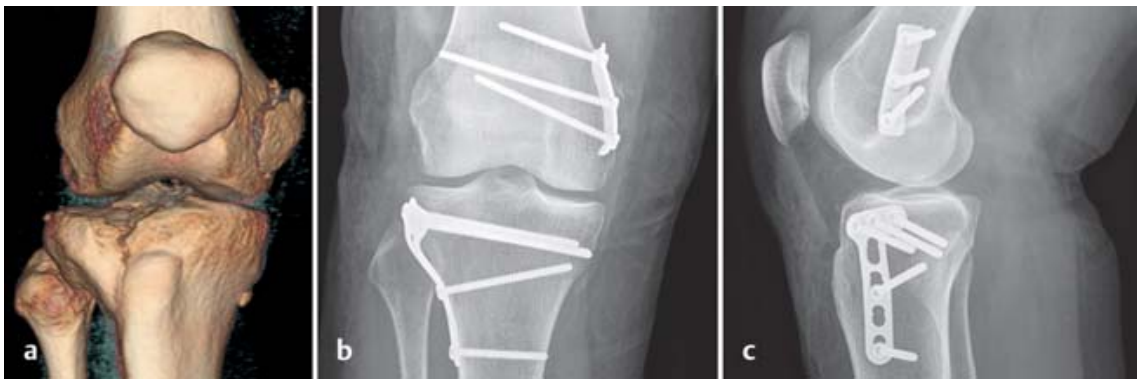

Abb. 1 a bis c Eine proximale, partiell artikuläre Tibiafraktur (AO 41-B3) mit zusätzlichem femoralem ossärem Ausriss des medialen Kollateralbands. a Initiales CT der Impressionsspaltfraktur. b, c Postoperative Versorgung mittels einer 3,5-mm-T-Platte in Abstützfunktion an der Tibia und $1 / 3$-Rohrplatte zur Fixation femoral. Winkelstabilität bei diesem jungen Patienten mit gutem Knochen nicht notwendig.

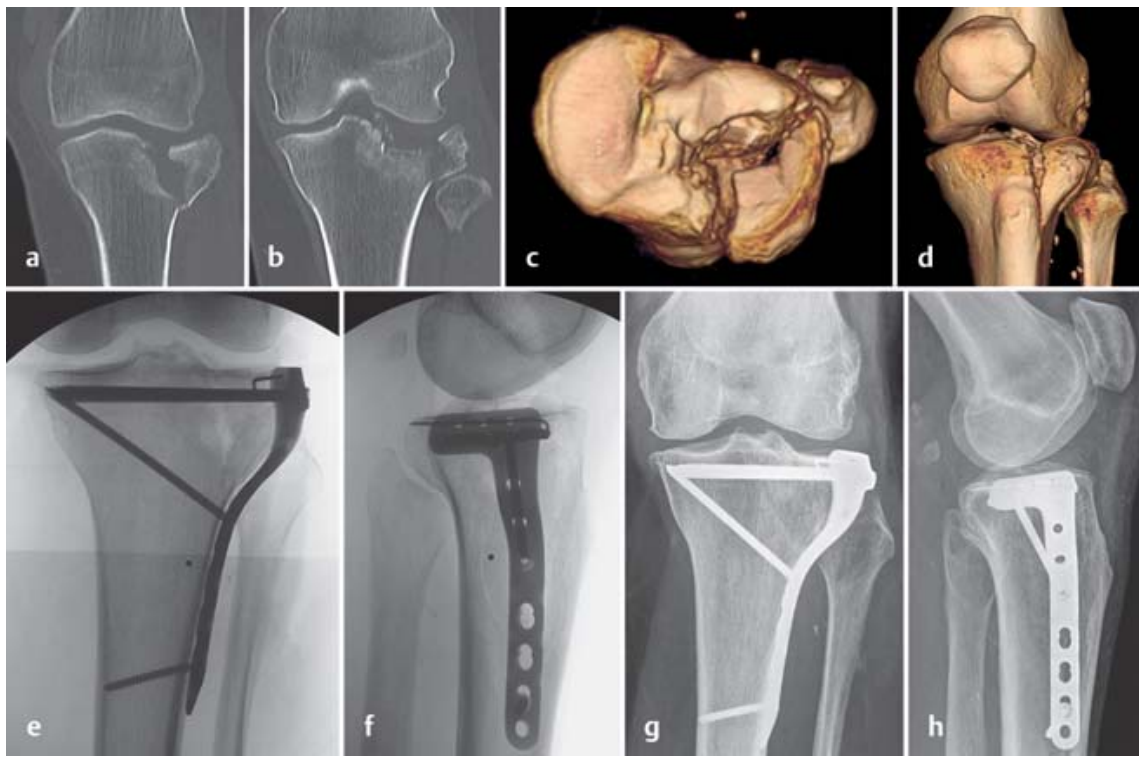

Abb. 2 a bis h a-d CT 2- und 3-D: 62-jährige Frau mit mehrfragmentärer lateraler Impressionsspaltfraktur des lateralen Plateaus (B3). e, f Röntgen postoperativ: Aufgrund der massiven Impression und der Osteoporose bietet die Winkelstabile gute Vorteile. Die anatomisch vorgeformte Platte ermöglicht ein Setzen der proximal verriegelten Schrauben sehr gelenksnah zur optimalen Unterstützung der rekonstruierten lateralen Gelenksfläche. g, h Heilungsbilder 1 Jahr nach Operation mit anatomisch geheiltem Plateau ohne Zeichen einer posttraumatischen Arthrose.
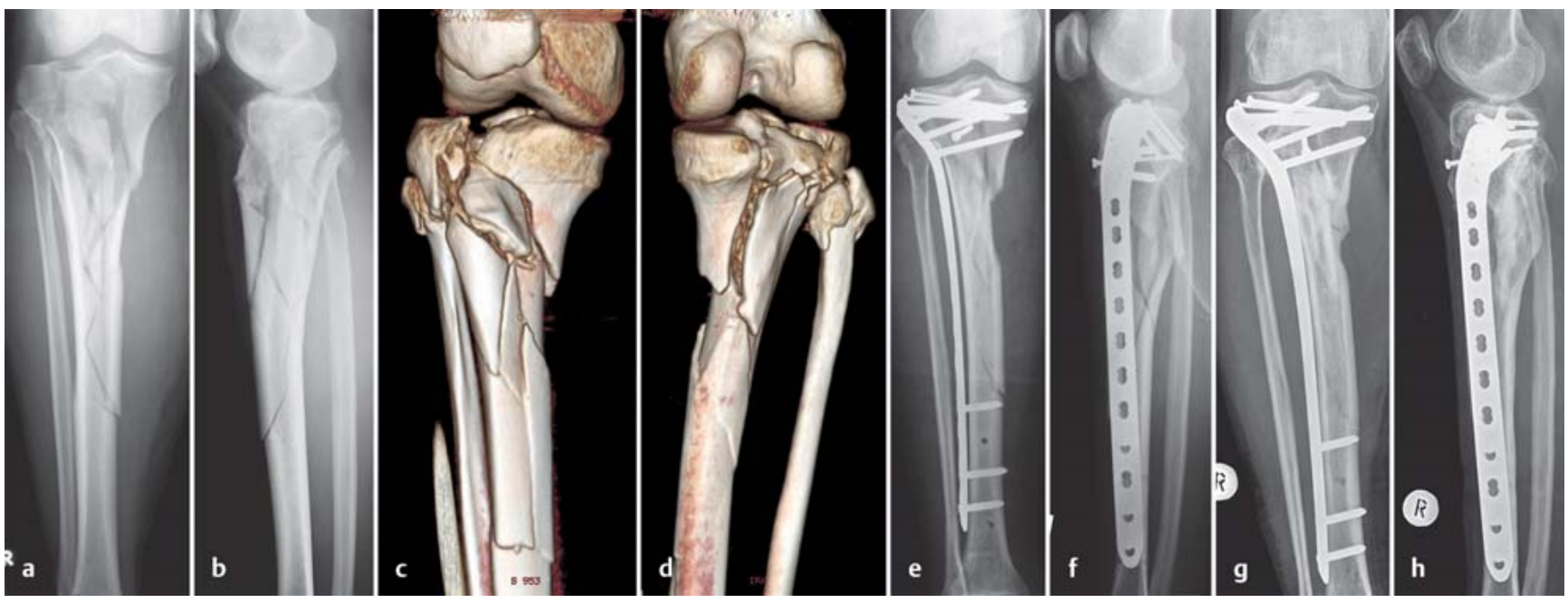

Abb. $\mathbf{3}$ a bis $\mathbf{h}$ a-d Komplett artikuläre Fraktur der proximalen Tibia (AO 41-C3) mit langstreckiger Ausdehnung in den Tibiaschaft. e-f Postoperative Stellung mit einer unilateralen winkelstabilen Versorgung der Fraktur (LCP-PLT 4,5/5,0). Die Gelenkskomponente ist mit zusätzlichen separaten, zuerst eingesetzten Zugschrauben 3,5 stabilisiert. Hier ist die Winkelstabilität essenziell. Alternativ käme eine bilaterale Verplattung infrage. g, $\mathbf{h} 7$ Monate postoperativ mit Heilung der Fraktur.

Yves Pascal Acklin et al.: Winkelstabile Plattenosteosynthese an der Tibia; 


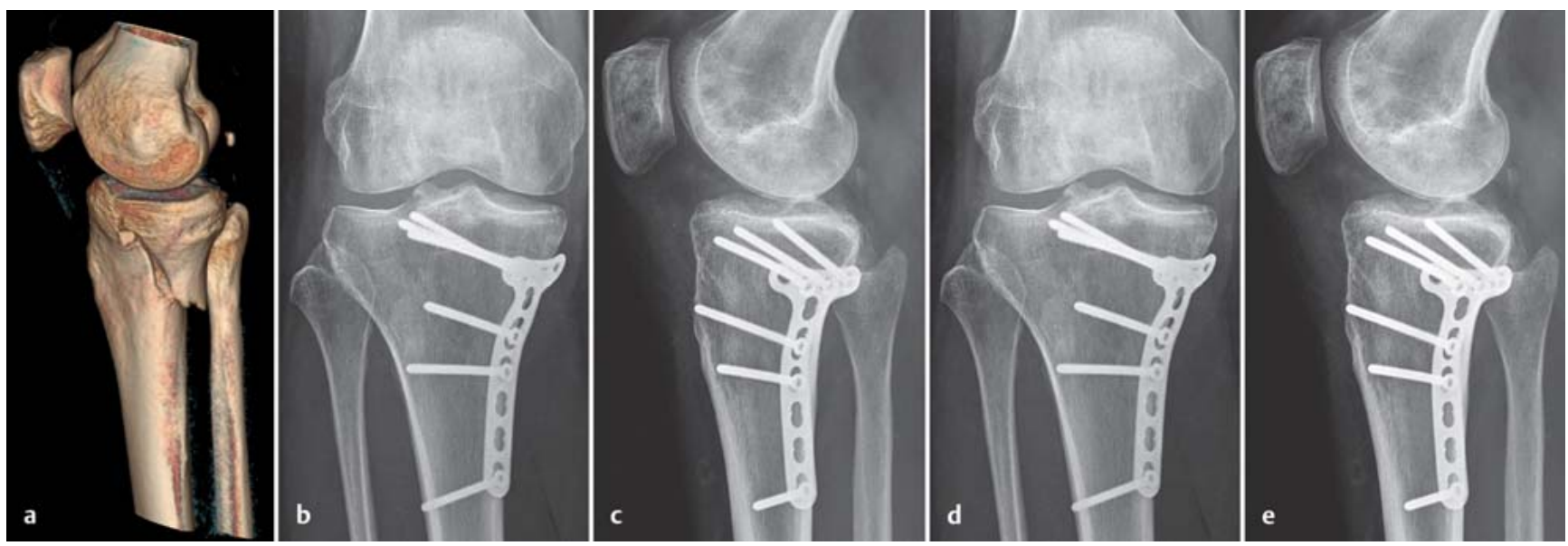

Abb. $\mathbf{4}$ a bis e Luxationfraktur Typ Moore II. a Das posteromediale Hauptfragment ist eindeutig zu erkennen. b, c Postoperative Röntgenaufnahme mit posteromedialer Antigleitplatte. d, e Heilungsbilder 1 Jahr postoperativ.

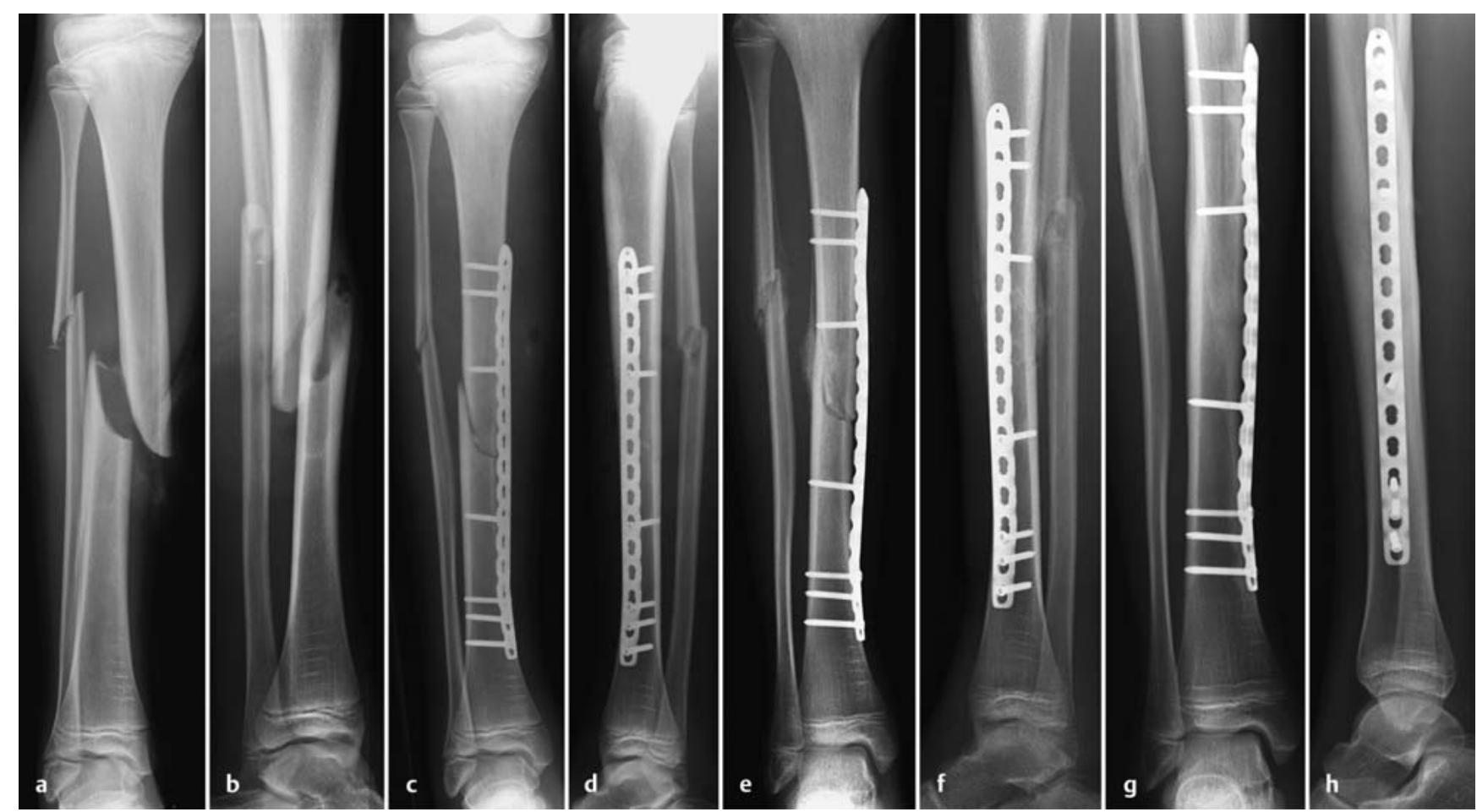

Abb. $\mathbf{5}$ a bis $\mathbf{h}$ Tibiaschaftfraktur. a, b Die Röntgenaufnahme zeigt eine erstgradige offene Tibiaschaftfraktur bei einem 15-jährigen Knaben mit noch offenen Epiphysenfugen. c, d Postoperative Versorgung mittels einer minimalinvasiv eingebrachten 3,5-mm-LCP. e-h Verlaufskontrolle 6 Wochen postoperativ und nach definitiver Heilung sowie Remodeling nach 9 Monaten.

\section{Winkelstabile Osteosyntheseverfahren bei distalen Tibiafrakturen}

Bei den distalen Tibiafrakturen ist vornehmlich die Unterscheidung in extraund intraartikuläre (Pilon-)Frakturen wichtig. Erstere können vollständig durch eine minimalinvasive Technik versorgt werden, bei den intraartikulären Frakturen hingegen ist die direkte Visualisierung der Gelenkskomponente notwendig. Bei den extraartikulären Frakturen kommt den winkelstabilen Implan- taten v.a. der mögliche Fixateur-interne-Effekt zugute. Auch bei diesen Frakturen ist somit die vollständige anatomische Anpassung der Platte nicht notwendig. Wichtig ist jedoch, dass die Platte im Bereich des Innenknöchels vollständig dem Knochen anliegt, um die Weichteilirritation durch das Implantat möglichst gering zu halten. Aus diesem Grund sind die neuen anatomisch vorgeformten Platten in diesem distalen Abschnitt auch entsprechend ausgedünnt und konvex entsprechend der Knochenform gebogen (Abb.6). Die Verankerung der Platte in winkelstabiler Technik ist auch in osteoporotischem Knochen von großem Vorteil [6].

Intraartikuläre Frakturen benötigen die direkte Visualisierung der Frakturzone.

Bei den Pilonfrakturen bietet die winkelstabile Plattenosteosynthese besonders bei komplexen Frakturmustern mit multiplen Fragmenten den Vorteil, eine sekundäre Dislokation der Gelenksfrag- 


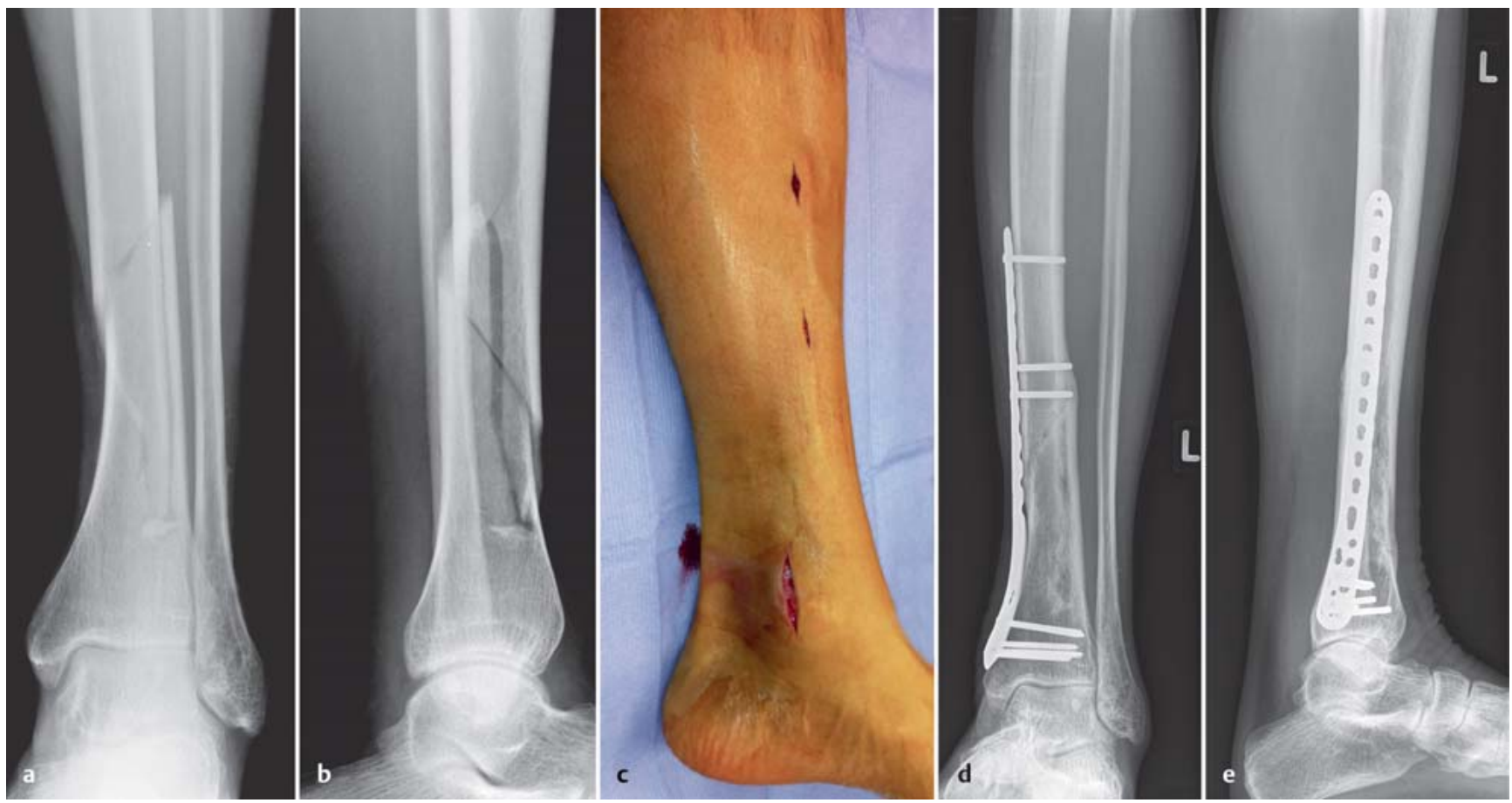

Abb. 6 a bis e Distale Tibiaschaftfraktur mit fissuralem Ausläufer ins Gelenk. a, b Radiologische Darstellung der Fraktur. c Minimalinvasiver Zugang zur Frakturversorgung. d, e Konsolidierte Fraktur 6 Monate nach Unfall.

mente durch die in der Platte fixierten Schrauben zu verhindern.

\section{Fazit}

Die winkelstabile Plattenosteosynthese bei Tibiafrakturen bietet nicht nur die Möglichkeit der minimalinvasiven Versorgung, sondern zeigt auch deutliche Vorteile in der Frakturstabilisierung im osteoporotischen Knochen im Vergleich zur herkömmlichen Platten-SchraubenVersorgung. Die neuen anatomisch vorgeformten Platten erleichtern die Versorgung sowohl proximaler wie auch distaler artikulärer Tibiafrakturen.

\section{Literatur}

${ }^{1}$ Sommer C, Gautier E, Muller M et al. First clinical results of the Locking Compression Plate (LCP). Injury 2003; 34 (Suppl. 2): B43-B54

2 Stoffel K, Dieter U, Stachowiak G et al. Biomechanical testing of the LCP - how can stability in locked internal fixators be controlled? Injury 2003; 34: 11-19

3 Cullen $A B$, Curtiss S, Lee MA. Biomechanical comparison of polyaxial and uniaxial locking plate fixation in a proximal tibial gap model. J Orthop Trauma 2009; 23: 507-513

4 Potocnik P, Acklin YP, Sommer C. Operative strategy in postero-medial fracture-dislocation of the proximal tibia. Injury 2011; 42: 1060-1065

${ }^{5}$ Bhandari M, Guyatt G, Tornetta 3rd P et al. Randomized trial of reamed and unreamed intramedullary nailing of tibial shaft fractures. J Bone Joint Surg [Am] 2008; 90: 2567-2578
${ }^{6}$ Snow M, Thompson G, Turner PG. A mechanical comparison of the locking compression plate (LCP) and the low contact-dynamic compression plate (DCP) in an osteoporotic bone model. J Orthop Trauma 2008; 22 : 121-125

\section{Dr. Dr. med. Yves Pascal Acklin} Oberarzt Orthopädie und Unfallchirurgie Dr. med. Christoph Sommer Leiter Unfallchirurgie

Kantonsspital Graubünden Loestr. 170 7000 Chur

Schweiz

yves.acklin@ksgr.ch 